PROCEEDINGS OF THE

AMERICAN MATHEMATICAL SOCIETY

Volume 130, Number 2, Pages 529-531

S 0002-9939(01)06109-3

Article electronically published on June 6, 2001

\title{
ASYMPTOTIC PROPERTIES \\ OF THE VECTOR CARLESON EMBEDDING THEOREM
}

\author{
MICHAEL GOLDBERG
}

(Communicated by Christopher D. Sogge)

\begin{abstract}
The dyadic Carleson embedding operator acting on $\mathbb{C}^{n}$-valued functions has norm at least $C \log n$. Thus the Carleson Embedding Theorem fails for Hilbert space valued functions.
\end{abstract}

Let $\mathbb{T}$ be the unit circle in $\mathbb{C}$, and $\{I\}_{I \in D}$ its collection of dyadic arcs. Let $w_{I}$ be nonnegative real numbers indexed by $I \in D$. For integrable functions $f$ on $\mathbb{T}$, denote by $\langle f\rangle_{I}$ the average $|I|^{-1} \int_{I} f(y) d y$. The classical Carleson embedding theorem [1] is equivalent to the following dyadic result:

Theorem. If $\sum_{I \subset K} w_{I} \leq|K|$ for all $K \in D$, then $\sum_{I \in D} w_{I}\langle f\rangle_{I}^{2} \leq C\|f\|^{2}$ for all $f \in L^{2}(\mathbb{T})$.

The converse is also true (up to the placement of constants) and is verified by considering functions of the form $f=\chi_{J}, J \in D$.

An analogous statement may be made for functions taking values in $\mathbb{C}^{n}$ with matrix-valued weights $W_{I} \geq 0$ in the sense of quadratic forms. We wish to consider the following $n$-dimensional embedding theorem:

Proposition. If $\left\|\sum_{I \subset K} W_{I}\right\| \leq|K|$ for all $K \in D$, then

$$
\sum_{I \in D}\left(W_{I}\langle f\rangle_{I},\langle f\rangle_{I}\right) \leq C_{n}\|f\|^{2}
$$

for all $f \in L^{2}\left(\mathbb{T} ; \mathbb{C}^{n}\right)$.

The space $\mathbb{C}^{n}$ here is viewed as a finite-dimensional Hilbert space. One might ask whether a similar result still holds when $f$ takes values in a general Hilbert space $\mathbb{H}$ and $W_{I}$ are positive selfadjoint operators. This is answered in the negative by [4], which proves that $C_{n}$ must be bounded from below by $c \log n$. In the current paper we will use the construction in [4] to verify the stronger bound $C_{n} \geq c(\log n)^{2}$, which is also proved in [5]. A precise statement is as follows:

Theorem 1. There exist a function $f \in L^{2}\left(\mathbb{T} ; \mathbb{C}^{n}\right)$ and matrix weights $W_{I} \geq 0$ such that $\left\|\sum_{I \subset K} W_{I}\right\| \leq|K|$ and $\sum_{I \in D}\left(W_{I}\langle f\rangle_{I},\langle f\rangle_{I}\right) \geq c(\log n)^{2}\|f\|^{2}$, where $c>0$ is independent of $n$.

Received by the editors July 5, 2000.

2000 Mathematics Subject Classification. Primary 42B20, 42 A50.

Key words and phrases. Carleson embedding theorem, vector valued functions, operator valued measures, weights. 
Remarks. The example presented here is due to Nazarov, Treil, and Volberg [4. It is further shown in [3] and [4] that the best possible $C_{n}$ is bounded above by $C(\log n)^{2}$, making these results sharp up to a constant factor.

Proof of Theorem 1. Let $\mathbf{e}_{0}, \mathbf{e}_{1}, \ldots, \mathbf{e}_{n}$ be the standard basis for $\mathbb{C}^{n+1}$. Define the Rademacher functions $r_{j}\left(e^{2 \pi i t}\right)=(-1)^{\left\lfloor 2^{j} t\right\rfloor}$. For a dyadic interval $I,|I| \leq 2^{-j}, r_{j}$ is seen to be constant along $I$. Its value throughout the interval will be called $r_{j}(I)$.

Let $f(x)=\sum_{j=0}^{n} r_{j}(x) \mathbf{e}_{j}$. Clearly $\|f\|^{2}=n+1$. The averages of $f$ over dyadic intervals are also easy to compute. When $|I|=2^{-i}, i \leq n,\langle f\rangle_{I}=\sum_{j=0}^{i} r_{j}(I) \mathbf{e}_{j}$.

Let $W_{I},|I| \geq 2^{-n}$, be the rank-one operator satisfying $W_{I} \mathbf{v}=|I|\left(\mathbf{v}, \phi_{I}\right) \phi_{I}$, where $\phi_{I}=\sum_{j=0}^{i} \frac{1}{i+1-j} r_{j}(I) \mathbf{e}_{j}$. Define $\phi_{I}$ to be 0 when $|I|<2^{-n}$. Already we can estimate the sum

$$
\sum_{I \in D}\left(W_{I}\langle f\rangle_{I},\langle f\rangle_{I}\right)=\sum_{I \in D}|I|\left(\langle f\rangle_{I}, \phi_{I}\right)^{2}=\sum_{i=0}^{n}\left(\sum_{j=0}^{i} \frac{1}{i+1-j}\right)^{2} \geq c n(\log n)^{2} .
$$

The only task remaining is to show that $\left\|\sum_{I \subset K} W_{I}\right\|$ is controlled by $|K|$. We will prove the estimate $\sum_{I \subset K}\left(W_{I} \mathbf{v}, \mathbf{v}\right)=\sum_{I \subset K}|I|\left(\mathbf{v}, \phi_{I}\right)^{2} \leq C|K||\mathbf{v}|^{2}$ for all $\mathbf{v} \in \mathbb{C}^{n+1}$.

For each interval $I$ with $|I|=2^{-i}$, split the vector $\phi_{I}$ into the sum of two parts, $\phi_{I}=\sum_{j=0}^{k} \frac{1}{i+1-j} r_{j}(K) \mathbf{e}_{j}+\sum_{j=k+1}^{i} \frac{1}{i+1-j} r_{j}(I) \mathbf{e}_{j}$. Denote the first sum, which depends only on the length of $I \subset K$, by $\mathbf{g}_{i}$. Summing over all $I$ with $|I|=2^{-i}$, and exploiting the orthogonality of the Rademacher functions,

$$
\sum_{\substack{I \subset K \\|I|=2^{-i}}}|I|\left(\mathbf{v}, \phi_{I}\right)^{2}=|K|\left(\left(\mathbf{v}, \mathbf{g}_{i}\right)^{2}+\sum_{j=k+1}^{i} \frac{1}{(i+1-j)^{2}}\left|\mathbf{v}_{j}\right|^{2}\right) .
$$

Thus

$$
\sum_{I \subset K}\left(W_{I} \mathbf{v}, \mathbf{v}\right)=|K|\left(\sum_{i=k}^{n}\left(\mathbf{v}, \mathbf{g}_{i}\right)^{2}+\sum_{j=k+1}^{n}\left|\mathbf{v}_{j}\right|^{2} \sum_{i=j}^{n} \frac{1}{(i+1-j)^{2}}\right) .
$$

The second sum is less than $C|K| \sum_{j=0}^{n}\left|\mathbf{v}_{j}\right|^{2}=C|K||\mathbf{v}|^{2}$. To estimate the first sum, let $\mathbf{G}$ represent the $(n-k+1) \times(k+1)$ matrix whose $i j^{\text {th }}$ entry is the coefficient of $\mathbf{e}_{j-1}$ in $\mathbf{g}_{i+k-1}$. Then $\sum_{i=k}^{n}\left(\mathbf{v}, \mathbf{g}_{i}\right)^{2} \leq\|\mathbf{G}\|^{2}|\mathbf{v}|^{2}$. Here $\|\mathbf{G}\|$ is taken as an operator from $\mathbb{C}^{k+1}$ to $\mathbb{C}^{n-k+1}$. Under a suitable permutation of indices, however, $\mathbf{G}$ is seen to be a restriction of the Hilbert matrix $\mathbf{A},\left.\left(\mathbf{A}_{i j}=\frac{1}{i+j-1}\right)\right|_{i, j=1} ^{\infty}$, to finite-dimensional subspaces. It is well known [2] that $\mathbf{A}$ is bounded from $\ell^{2}(\mathbb{N})$ to itself. Thus the first sum is less than $|K|\|\mathbf{A}\|^{2}|\mathbf{v}|^{2}=C|K||\mathbf{v}|^{2}$. Dividing all weights $W_{I}$ by an appropriate constant proves the theorem.

\section{REFERENCES}

[1] J. B. Garnett, Bounded Analytic Functions, Acad. Press, New York, 1981. MR 83g:30037

[2] G. H. Hardy, J. E. Littlewood, and G. Polya, Inequalities, University Press, Cambridge, 1934.

[3] Nets Hawk Katz. Matrix valued paraproducts, J. Fourier Anal. Appl. 300 (1997), pp. 913-921. MR 99f: 42046 
ASYMPTOTIC PROPERTIES OF THE VECTOR CARLESON EMBEDDING THEOREM 531

[4] F. Nazarov, S. Treil, and A. Volberg, Counterexample to the infinite dimensional Carleson embedding theorem, C.R. Acad. Sci. Paris Sér. I Math., 325 (1997), No.4, pp. 383-388. MR 98d:46039

[5] F. Nazarov, G. Pisier, S. Treil, and A. Volberg, Sharp estimates in the vector Carleson imbedding theorem and for vector paraproducts, Preprint.

Department of Mathematics, University of California, Berkeley, California 947203840

E-mail address: mikeg@math.berkeley.edu 